\title{
PERÍODO DE RECRUTAMENTO E USO DE RECURSOS ESPACIAIS POR Tropidurus hygomi (SAURIA: TROPIDURIDAE) EM UMA ÁREA DE ECÓTONO, NORDESTE, BRASIL
}

\author{
Tainara Lima-Silva ${ }^{1^{*}}$, Maria Aldenise Xavier ${ }^{2}$ \& Eduardo José dos Reis Dias ${ }^{1}$
}

\begin{abstract}
${ }^{1}$ Universidade Federal de Sergipe, Departamento de Biociências, Laboratório de Biologia e Ecologia de Vertebrados, Av. Olímpio Grande, s/n, Campus Professor Alberto Carvalho, CEP 49500-000, Itabaiana, SE, Brasil.

${ }^{2}$ Universidade Federal da Bahia, Departamento de Zoologia, Programa de Pós-Graduação em Biodiversidade e Evolução. Rua Barão de Jeremoabo, s/n, Campus Universitário de Ondina, CEP 40170-115, Salvador, BA, Brasil.
\end{abstract}

E-mails: tainara.lima2@outlook.com (*autor correspondente); aldenisexavier@hotmail.com; ejrdias@hotmail.com

Resumo: O uso do microhabitat pode ter um efeito direto na sobrevivência dos lagartos através de comportamentos de forrageio, termorregulação, dieta e atividade diária, utilizados por cada espécie, influenciando no modo como eles obtêm seus recursos no ambiente. Assim, este estudo objetivou analisar as diferenças ontogenéticas quanto ao uso do microhabitat e o período de recrutamento de Tropidurus hygomi no Parque Nacional Serra de Itabaiana, estado de Sergipe, Brasil. Os dados foram coletados mensalmente, no período entre 2015 e 2016, e os lagartos foram contabilizados e diferenciados em jovens e adultos a cada intervalo de hora (09:00 - 15:00 h). Os microhabitats utilizados por jovens e adultos foram categorizados a priori. Tropidurus hygomi apresentou um período de recrutamento contínuo, mas com uma leve variação ao longo das estações, pois jovens foram registrados em todo o período amostrado. Jovens e adultos não apresentaram diferença quanto a sua abundância e a utilização dos microhabitats, e apresentaram uma alta sobreposição espacial. Essa sobreposição espacial encontrada entre as faixas etárias pode ser explicada pela qualidade dos habitats, com áreas mais sombreadas para evitar o superaquecimento.

Palavras-chave: nicho espacial; temperatura; uso de microhabitat; vegetação.

\section{RECRUITMENT PERIOD AND SPATIAL RESOURCE USES OF Tropidurus hygomi (SAURIA:TROPIDURIDAE)} IN ECOTONE AREA, NORTHEAST, BRAZIL The microhabitat use can have a direct effect on the survival of the lizards through foraging, thermoregulation, diet and daily activity behaviors used by each species, influencing how they obtain their resources in the environment. Thus, this study aimed to analyze the ontogenetic differences concerning the microhabitat use and recruitment period of Tropidurus hygomi in the Parque Nacional Serra de Itabaiana, Sergipe state, Brazil. The data were collected monthly between 2015 and 2016, and the lizards were counted and differentiated in juveniles and adults at each hour interval 09:00 - 15:00 h). The microhabitats used by juveniles and adults were categorized a priori. Tropidurus hygomi presented a continuous recruitment period, but with a slight variation throughout the seasons, since juveniles were recorded throughout the sampled period. Juveniles and adults did not present differences regarding their abundance and the microhabitats use, and presented a high spatial overlap. This spatial overlap between the age groups can be explained by the quality of the habitats, with more shaded areas to avoid overheating.

Keywords: spatial niche; temperature; use of microhabitat; vegetation. 


\section{INTRODUÇÃO}

O uso do microhabitat pode ter um efeito direto na sobrevivência dos lagartos (Van Sluys 1993, Wiederhecker et al. 2002), dessa forma, alguns comportamentos como forrageamento, termorregulação, dieta e atividade diária, utilizados por cada espécie, influenciam no modo como eles obtêm seus recursos no ambiente, e constituem alguns dos aspectos mais importantes da ecologia desses organismos (Vitt 1991, Colli et al. 1992, Meira et al. 2007). A dinâmica de uso do espaço pode também ser influenciada por fatores como coexistência intraespecífica e variação sazonal nas condições ambientais (Salzburg 1984, Paulissen 1988). Sendo assim, alguns autores argumentam que o uso do microhabitat por lagartos, muitas vezes, pode diferir entre diferentes faixas etárias e tamanho corporal (CRC) em uma população, o que pode ser atribuído ao comportamento territorialista (comum em Tropiduridae) ou a escolha do local de forrageio (Marcellini \& Mackey 1970, Gibbons \& Lillywhite 1981, Paulissen 1988, Rocha et al. 2000).

O gênero Tropidurus é caracterizado por possuir espécies que possuem ocorrência ampla em áreas abertas e florestais do Brasil (Rodrigues 1988), onde suas populações são localmente abundantes (Van Sluys 2000). Espécies desse gênero apresentam diferentes padrões reprodutivos, podendo ser contínuo ao longo do ano ou sazonal, que podem ter uma relação direta com os fatores climáticos, principalmente, com a pluviosidade (Rocha 1992, 1998, Vitt 1993, Wiederhecker et al. 2002, Galdino et al. 2003, Meira et al. 2007, Balestrin et al. 2010, Ribeiro et al. 2012).

Atualmente, há um considerável conhecimento sobre a história natural (e.g., comportamento, distribuição geográfica, padrão reprodutivo, período de atividade, hábito alimentar, modo de forrageamento e termorregulação) do gênero Tropidurus no Brasil (e.g., Rodrigues 1988, Teixeira \& Giovanelli 1999, Meira et al. 2007, Vargens et al. 2008, Martins et al. 2010, Vieira et al. 2011, Ribeiro et al. 2012, Xavier \& Dias 2017), porém, existe a necessidade de reforçar o conhecimento sobre o período de recrutamento e as relações ontogenéticas desse grupo quanto ao uso de microhabitat.

Tropidurus hygomi Reinhardt \& Lutken, 1861 é um lagarto heliófilo, restrito a ambientes com solo arenoso, distribuído em áreas de restinga, desde o Litoral Norte da Bahia até o Litoral Norte do estado de Sergipe (Dias \& Rocha 2014, Xavier \& Dias 2015), mas também apresenta uma população isolada no interior do estado de Sergipe, no Parque Nacional Serra de Itabaiana (PNSI) (Rodrigues 1988, Carvalho 2013). Esta espécie de lagarto apresenta um comprimento rostro-cloacal de aproximadamente $50 \mathrm{~mm}$, sendo considerada insetívora, territorialista e com baixa circulação entre moitas (Vargens et al. 2008, Martins et al. 2018). Ainda, recentemente a espécie foi categorizada como vulnerável (VU A2bc) no Livro Vermelho da Fauna Brasileira Ameaçada de Extinção (MMA 2016).

Considerando a influência de diversos fatores ecológicos quanto ao uso do microhabitat, este estudo tem como hipóteses: 1) os jovens e adultos de T. hygominão compartilham o mesmo microhabitat; e 2) a espécie T. hygomi apresenta um período de recrutamento sazonal. Sendo assim, o principal objetivo deste estudo foi avaliar as diferenças quanto ao uso de microhabitat entre jovens e adultos e o período de recrutamento de jovens em uma população de T. hygomi no Parque Nacional Serra de Itabaiana, estado de Sergipe, Brasil.

\section{MATERIAL E MÉTODOS}

O presente estudo foi realizado no PNSI (37²5'15”O, $10^{\circ} 25^{\prime} 15^{\prime \prime}$, datum WGS84), localizado no município de Areia Branca, estado de Sergipe, Brasil (Figura 1). O PNSI apresenta uma área de aproximadamente oito mil hectares, composta por diferentes ambientes como áreas abertas com areias brancas e vegetação de Agreste, e áreas de formações ombrófila densa (Bezerra et al. 2007), considerada como uma região ecotonal. Segundo a classificação climática de Köppen-Geiger, o clima da região é classificado como tropical com verão seco ("As") (Peel et al. 2007). A temperatura média local é de $24,1^{\circ} \mathrm{C}$, umidade relativa do ar de $63,9 \%$ e a precipitação média anual de $1300 \mathrm{~mm}$ (ClimateData.Org.), as quais variam ao longo dos meses, como descrito no diagrama climático da área no período de amostragem (2015-2016) (Figura 2).

As áreas de areias brancas do PNSI apresentam uma fisionomia semelhante às restingas sergipanas, por exibir um solo branco quartzoso exposto e uma vegetação arbustiva esclerófila (Dantas \& Ribeiro 2010). Apesar de não serem 


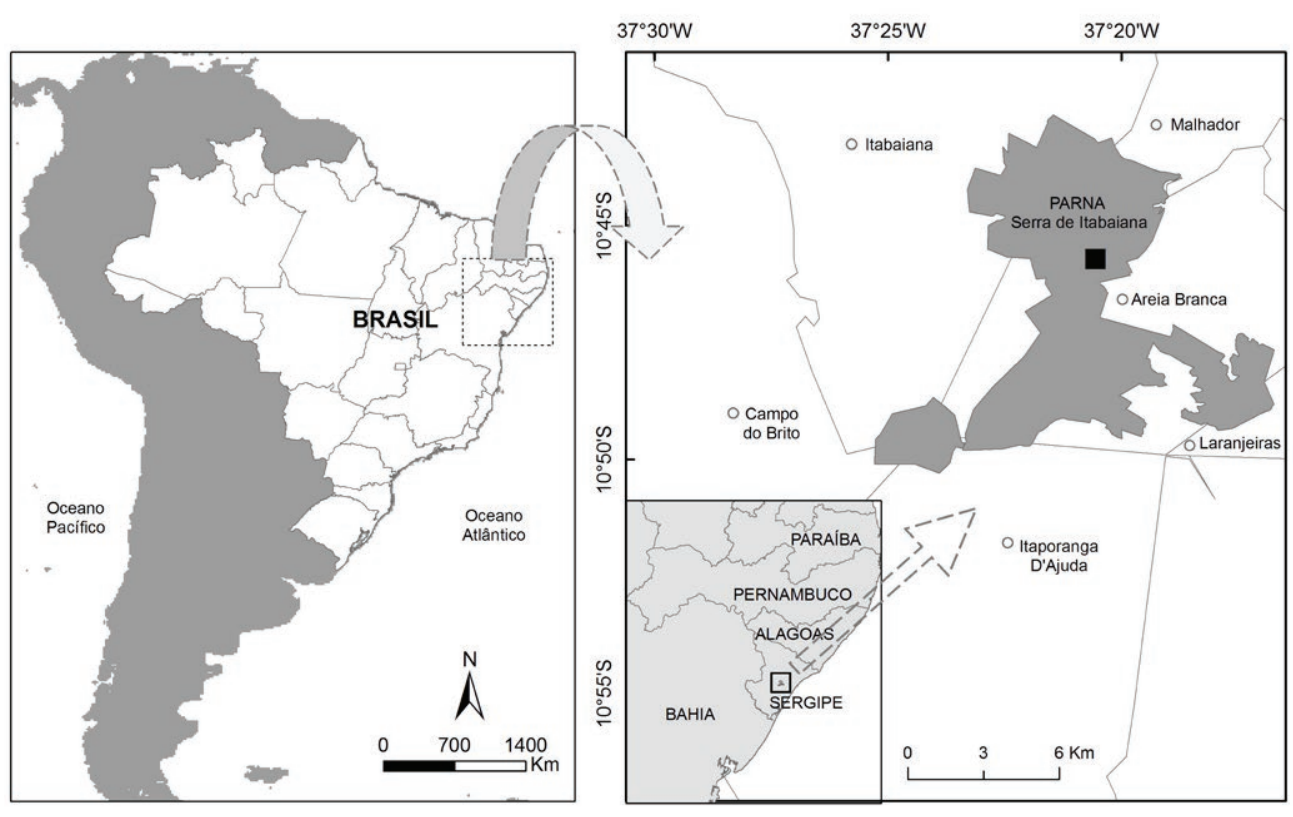

Figura 1. Localização geográfica da área de estudo no Parque Nacional Serra de Itabaiana, estado de Sergipe, Brasil.

Figure 1. Geographic location of the study area in the Parque Nacional Serra de Itabaiana, Sergipe state, Brazil.

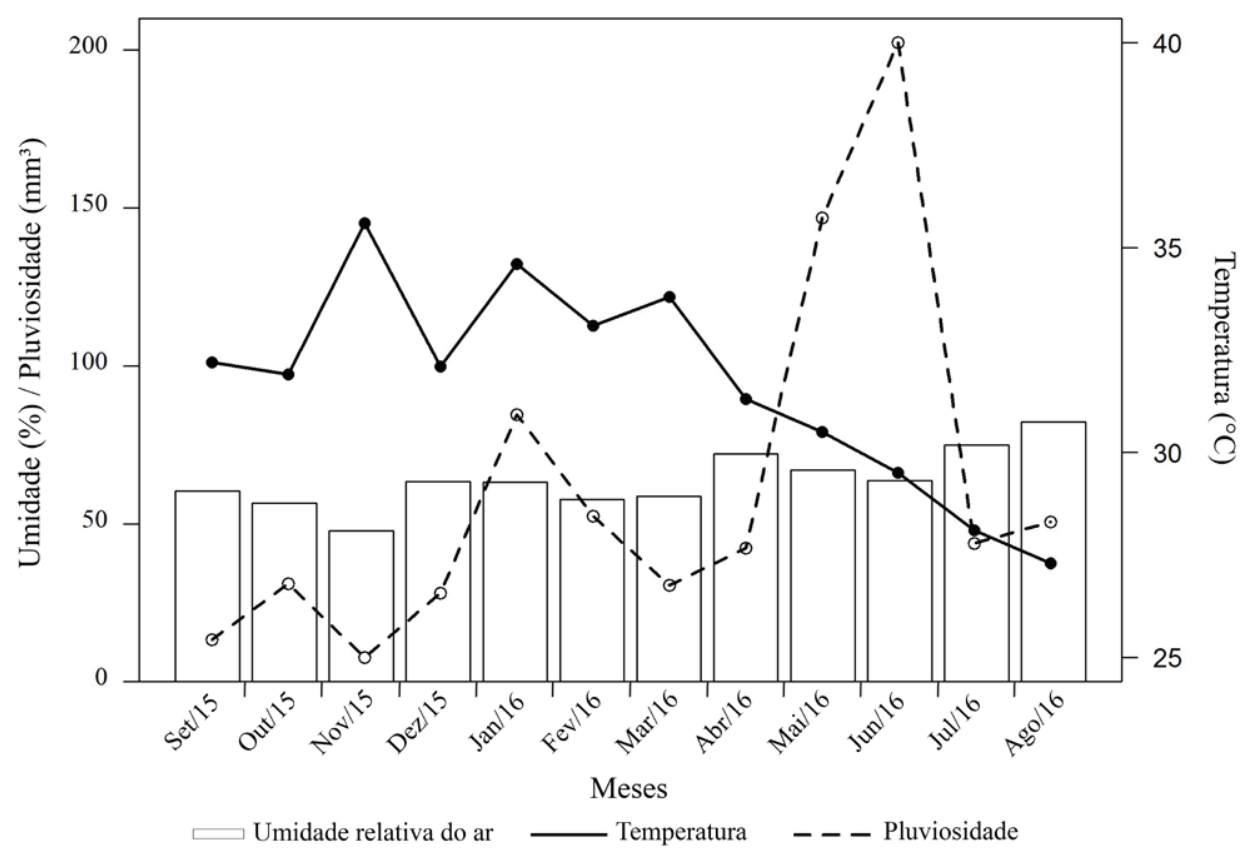

Figura 2. Diagrama climático com dados médios de temperatura e umidade do ar, e pluviosidade para o Parque Nacional Serra de Itabaiana (PNSI) no período de amostragem. Os dados de pluviosidade foram da estação meteorológica mais próxima do PNSI, no período de 2015-2016, extraídos do Instituto Nacional de Meteorologia (INMET).

Figure 2. Climatic diagram with average data of air temperature and humidity, and rainfall for the Parque Nacional Serra de Itabaiana (PNSI) in the sampling period. The rainfall data were the nearest meteorological station of the PNSI, in the period 2015-2016, extracted from the National Institute of Meteorology (INMET). 
ambientes de restinga, elas são consideradas como um Refúgio Ecológico, com vegetação azonal derivada de condições especiais de formação do substrato (IBGE 1992, Dantas \& Ribeiro 2010, Dantas et al. 2010). A estrutura vegetacional da área de estudo é caracterizada por um estrato arbóreo ralo e disperso, com uma altura média de 4,6 $\pm 2,1$ m (Áreas de areias brancas abertas) e 4,0 $\pm 1,2 \mathrm{~m}$ (Áreas de areias brancas fechadas) (Dantas et al. 2010). O dossel é composto por arbustos entre três e seis metros, raramente com árvores acima de sete metros. Os subarbustos são isolados em regiões de solo desnudo com espécies micrófilas esclerófilas e o estrato herbáceo ocorre de forma dispersa entre os arbustos ou em solos sem cobertura vegetal (Bezerra et al. 2007, Dantas \& Ribeiro 2010, Dantas et al. 2010).

A amostragem foi realizada mensalmente, durante dois dias consecutivos, ao longo de um ano (setembro de 2015 a agosto de 2016). Para acessar a informação do uso do microhabitat e o período de recrutamento de jovens foi selecionada uma área de $60.000 \mathrm{~m}^{2}$, dividida em seis parcelas (100 x $100 \mathrm{~m})$ equidistantes $(100 \mathrm{~m})$. As parcelas foram amostradas durante $20 \mathrm{~min}$, a cada intervalo de hora, das 09:00 às 15:00 $\mathrm{h}$, com esforço amostral de três pessoas, totalizando $144 \mathrm{~h}$ de busca. Cada ponto de coleta foi amostrado em diferentes períodos do dia, evitando repetições de horários.

O microhabitat onde cada lagarto se encontrava no momento do primeiro avistamento foi registrado e categorizado em: (1) Areia nua fora de moita; (2) Sob vegetação herbácea com folhiço e bromélia; (3) Sob vegetação herbácea sem folhiço e bromélia; (4) Sob vegetação arbustiva com folhiço e bromélia; e (5) Sob vegetação arbustiva sem folhiço e bromélia. Para cada ponto de coleta foram registradas as alturas da vegetação com uma trena (precisão de $1 \mathrm{~cm}$ ) e arbustos acima de $2 \mathrm{~m}$ por estimativa visual (Curto et al. 2013). Foram mensuradas a temperatura e a umidade relativa do ar com uma estação meteorológica (Kestrel ${ }^{\circledR} 4300$ ) e a temperatura do substrato com um termômetro digital infravermelho $\left(\mathrm{Akso}^{\circledR} \mathrm{AK} 30\right)$, em cada intervalo de hora.

Os lagartos foram contabilizados, diferenciando os jovens ( $\mathrm{CRC}<50,8 \mathrm{~mm}$ ) dos adultos. A distinção da faixa etária foi feita a partir do tamanho médio proposto por Lima (2016) em seu trabalho de histologia, o qual determina o tamanho mínimo de maturação para os machos e fêmeas de T. hygomi na mesma área de estudo.

Para uma melhor compreensão da influência das estações climáticas no recrutamento de jovens, o período de amostragem de dados em campo foi dividido em estação seca (S), compreendendo os meses de setembro a fevereiro (S1 a S6, respectivamente) e estação chuvosa (C) que compreende os meses de março a agosto (C1 a C6, respectivamente).

Oteste de Kolmogorov-Smirnovfoi utilizado para avaliar as diferenças quanto ao uso do microhabitat entre jovens e adultos. Para avaliar a diferença entre as duas classes etárias quanto ao uso da altura de vegetação foi utilizado o teste de Mann-Whitney (Zar 2010). Uma Análise de Componentes Principais (ACP) utilizando os meses das estações seca e chuvosa como objetos e as variáveis climáticas temperatura do ar, pluviosidade e umidade relativa do ar (padronizadas a priori) como descritores, foi realizada a fim de verificar o efeito dessas variáveis ambientais nas duas estações (Zar 2010). O teste $t$ de student foi utilizado para avaliar a diferença na abundância de jovens e adultos de T. hygomi ao longo do ano (Zar 2010). Para avaliar a relação entre a abundância de jovens e adultos com as médias de temperatura e precipitação mensais foi utilizada a análise de Regressão simples (Zar 2010). Já o Índice de Diversidade de Simpson (Simpson 1949) foi usado para estimar a largura de nicho espacial, e o Coeficiente de Simetria de Sobreposição de Pianka (Pianka 1986) para avaliar as sobreposições de nicho espacial, ambos entre jovens e adultos. As premissas estatísticas foram testadas a partir do teste Shapiro-Wilk's (Shapiro \& Wilk 1965). $\mathrm{O}$ valor de significância adotado foi $\mathrm{p}<0,05$. As análises estatísticas e gráficas foram realizadas nos Softwares R (v3.4.2 R Development Core Team 2017), STATISTICA 8.0 e BioEstat 5.0.

\section{RESULTADOS}

Um total de 1613 indivíduos foram avistados em $144 \mathrm{~h}$ de busca, realizadas durante o período amostrado, dos quais $46,7 \%$ foram jovens e $53,3 \%$ adultos. Jovens e adultos de T. hygomi estiveram ativos com abundâncias semelhantes durante todos os horários amostrados neste estudo (Figura 3). O microhabitat com a maior frequência de indivíduos de T. hygomi foi a vegetação arbustiva com folhiço 
e bromélia (Adultos: 83,9\%, $\mathrm{N}=756$; Jovens: $65,2 \%$, $\mathrm{N}=510$ ). Tanto os jovens quanto os adultos foram observados utilizando outros tipos de microhabitats (Tabela 1). Não houve diferença estatisticamente significativa entre as duas faixas etárias quanto ao uso dos microhabitats $\left(\mathrm{D}_{\max }=0,187 ; \mathrm{p}=0,086\right)$. A largura do nicho espacial dos adultos $\left(B_{i j}=1,4\right)$ foi menor do que a dos jovens $\left(B_{i k}=2,9\right)$ e a sobreposição de nicho foi relativamente alta $(0,92)$.

A mediana da altura da vegetação dos microhabitats utilizados pelos jovens foi inferior (Mediana: $2 \mathrm{~m}$; amplitude: 0,2 - $10 \mathrm{~m}$ ) comparado com os adultos (Mediana: 2,2 m; amplitude: $0,1-10 \mathrm{~m}$ ), e essa diferença foi estatisticamente significativa $(U=6,319 ; \mathrm{p}<0,001)$. Os valores médios das variáveis ambientais durante a estação seca foram: umidade relativa do ar $(58,1 \pm 5,8 \%)$, temperatura do ar $\left(33,2 \pm 1,5^{\circ} \mathrm{C}\right)$ e precipitação $\left(36,3 \pm 28,9^{\circ} \mathrm{C}\right)$. E durante a estação chuvosa foram: umidade relativa do ar $(69,8 \pm 8,4 \%)$, temperatura do $\operatorname{ar}\left(30,1 \pm 2,3^{\circ} \mathrm{C}\right)$ e precipitação $\left(87,1 \pm 72,4^{\circ} \mathrm{C}\right)$.

A ACP aplicada às variáveis ambientais gerou três componentes principais, sendo que $66,6 \%$ da variação dos dados foi explicada pelo primeiro eixo (temperatura e umidade) e $28,8 \%$ pelo segundo eixo (pluviosidade) (Tabela 2). A projeção dos dois primeiros eixos da ACP agrupou os meses da estação seca e chuvosa (Figura 4). A baixa quantidade de chuva e de umidade relativa do ar, e a alta temperatura do mês de março (C1), devido o período de estudo ter sido atípico quanto à pluviosidade, agrupou-o aos meses da estação seca. A estação chuvosa foi melhor representada pelos meses de maio (C3) e junho (C4), apresentando maiores valores de pluviosidade e umidade relativa do ar, e baixa temperatura (Figura 2).

Os jovens de T. hygomi foram registrados ao longo de todo o ano, com maiores abundâncias no início da estação seca (setembro e outubro) e no final da estação chuvosa (junho a agosto), que correspondeu aos meses de menor temperatura (Figura 5). Os adultos acompanharam o aumento da pluviosidade, com maior abundância na estação chuvosa (abril, maio e junho), e não estavam diretamente relacionados com a temperatura. $\mathrm{O}$ número de adultos foi superior aos dos jovens entre os meses de dezembro a maio (Figura 5), mas não houve diferença significativa na abundância de jovens e adultos de T. hygomi durante o período amostrado $\left(\mathrm{t}_{1,22}=-0,498 ; \mathrm{g} .1 .=22 ; \mathrm{p}=0,688\right)$.

A média de lagartos jovens foi de $33,5 \pm 25,0$ na estação seca e de 44,0 $\pm 29,7$ na estação chuvosa

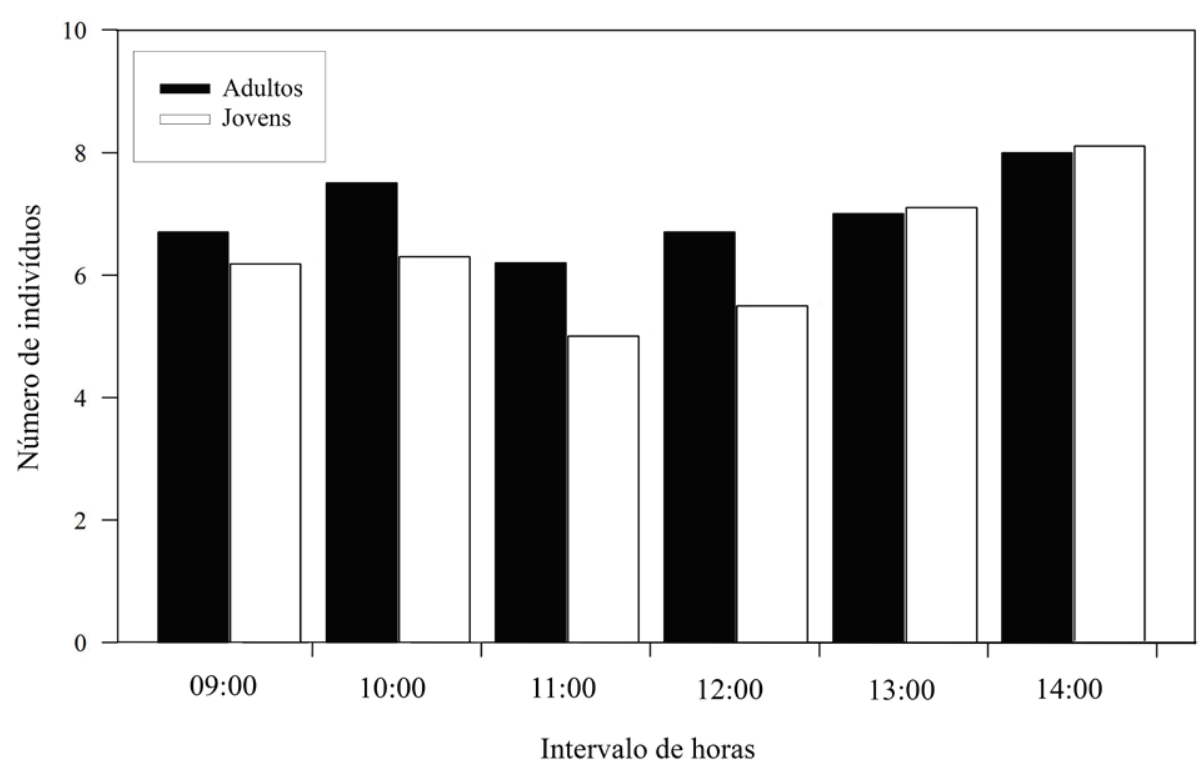

Figura 3. Abundância média de jovens e adultos de Tropidurus hygomi (Sauria, Tropiduridae) ao longo das horas de amostragem no Parque Nacional Serra de Itabaiana, estado de Sergipe, Brasil.

Figure 3. Mean abundance of young and adults of Tropidurus hygomi (Sauria, Tropiduridae) throughout the sampling hours in the Parque Nacional Serra de Itabaiana, Sergipe state, Brazil. 
(Figura 5). O número de jovens de T. hygomi não foi relacionado com a pluviosidade $\left(\mathrm{R}^{2}=0,118 ; \mathrm{F}_{1,10}\right.$ $=1,342 ; \mathrm{p}=0,274)$, mas apresentou relação com a temperatura média mensal $\left(R^{2}=0,448 ; F_{1,10}=8,115\right.$; $\mathrm{p}=0,017$ ) (Figura 5). O maior número de adultos foi registrado na estação chuvosa $(55,1 \pm 23,1)$ quando comparado com a estação seca $(32,1 \pm 10,3)$, sendo explicado pela pluviosidade $\left(\mathrm{R}^{2}=0,634 ; \mathrm{F}_{1,10}=17,35\right.$; $\mathrm{p}=0,002)$, mas não pela temperatura média mensal $\left(R^{2}=0,053 ; F_{1,10}=0,559 ; p=0,472\right)$ (Figura 5).

\section{DISCUSSÃO}

O lagarto T. hygomi é considerado uma espécie generalista quanto ao uso do microhabitat, pois ocupa ambientes com diversas características estruturais (e.g., diversas categorias de microhabitat, alturas de vegetação e frequência de regiões com pouca ou nenhuma cobertura vegetal) como encontrado por Vargens et al. (2008) e Martins et al. (2010) para essa mesma espécie no ecossistema restinga. Neste estudo, jovens e adultos de $T$. hygomi utilizaram microhabitats com vegetação arbustiva, contendo folhiço e bromélia em sua base, apresentando relativamente uma alta sobreposição de nicho $(0,92)$. Essa sobreposição pode ser explicada pela estrutura da vegetação da área de estudo, caracterizada por baixo dossel e vegetação esparsa, semelhante ao ecossistema restinga (Hatano et al. 2001). Esse tipo de estrutura pode fornecer uma grande área ensolarada durante o dia, levando aos lagartos procurarem áreas mais sombreadas para evitar superaquecimento (Grover 1996).

Embora a abundância de jovens e adultos tenha sido semelhante ao longo do período de amostragem, os jovens apresentaram maior largura de nicho em relação aos adultos. Esse fato pode estar relacionado à elevada taxa de movimentação dos jovens para encontrar e estabelecer territórios (Rocha et al. 2000, Vitt 2000), e aos fatores bióticos, como a competição interespecífica (Salzburg 1984, Grover 1996). Os lagartos de diferentes tamanhos podem utilizar estratégias comportamentais ou habitats distintos devido aos diferentes riscos de

Tabela 1. Porcentagem de jovens e adultos de Tropidurus hygomi (Sauria, Tropiduridae) em diferentes categorias de microhabitats no Parque Nacional Serra de Itabaiana, estado de Sergipe, Brasil.

Table 1. Percentage of juveniles and adults of Tropidurus hygomi (Sauria, Tropiduridae) in different microhabitat categories in the Parque Nacional Serra de Itabaiana, Sergipe state, Brazil.

\begin{tabular}{ccc}
\hline Categorias de microhabitats & Jovens & Adultos \\
\hline Areia nua fora de moita & 16,8 & 7,1 \\
\hline Sob vegetação herbácea com folhiço e bromélia & 3,1 & 0,8 \\
\hline Sob vegetação herbácea sem folhiço e bromélia & 9,0 & 2,1 \\
\hline Sob vegetação arbustiva com folhiço e bromélia & 65,2 & 83,9 \\
\hline Sob vegetação arbustiva sem folhiço e bromélia & 6,0 & 6,1 \\
\hline
\end{tabular}

Tabela 2. Coeficientes padronizados da Análise de Componentes Principais para três variáveis ambientais de 12 unidades amostrais para o Município de Areia Branca, estado de Sergipe, Brasil. Os escores dos componentes estão projetados na Figura 3.

Table 2. Standard Coefficients of Principal Component Analysis for three environmental variables of 12 sample units for the municipality of Areia Branca, Sergipe state, Brazil. The component scores are shown in Figure. 3.

\begin{tabular}{cccc}
\hline Variáveis ambientais & PC1 & PC2 & PC3 \\
\hline Pluviosidade & 0,515 & 0,856 & $-0,050$ \\
Temperatura & $-0,952$ & 0,149 & $-0,269$ \\
Umidade & 0,910 & $-0,328$ & $-0,253$ \\
Eigenvalue & 1,999 & 0,863 & 0,138 \\
Variância total (\%) & 66,6 & 28,8 & 4,6 \\
\hline
\end{tabular}




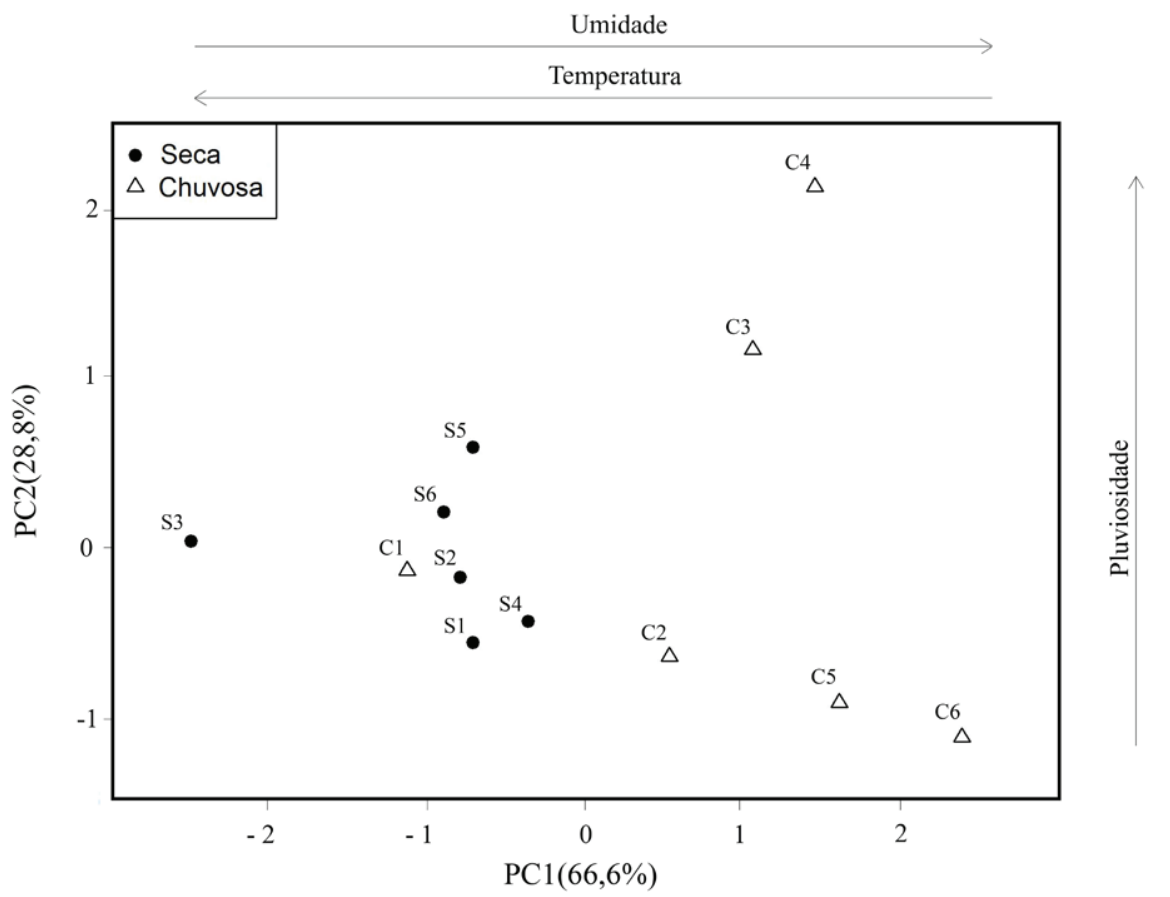

Figura 4. Projeção dos escores individuais resultantes da Análise de Componentes Principais para as variáveis ambientais temperatura e umidade do ar (PC1) e pluviosidade (PC2).

Figure 4. Projection of the individual scores resulting from the Principal Component Analysis for the environmental variables air temperature and humidity (PC1) and rainfall (PC2).

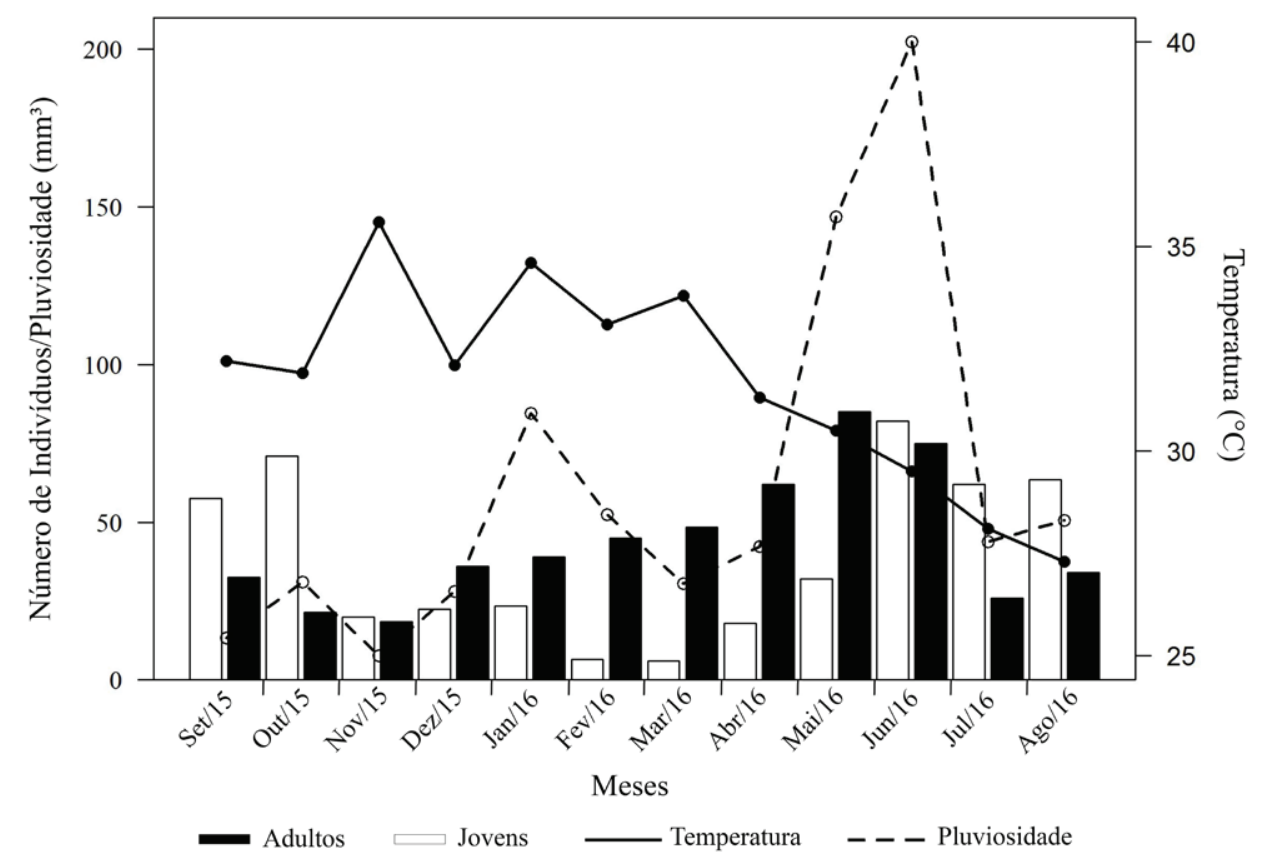

Figura 5. Relação dos dados de pluviosidade $(\mathrm{mm})$ e temperaturas médias mensais $\left({ }^{\circ} \mathrm{C}\right)$ com o número de jovens e adultos (período de recrutamento) de Tropidurus hygomi (Sauria, Tropiduridae) no Parque Nacional Serra de Itabaiana, Estado de Sergipe, Brasil.

Figure 5. Relation of rainfall data $(\mathrm{mm})$ and average monthly temperatures $\left({ }^{\circ} \mathrm{C}\right)$ with the number of juveniles and adults (recruitment period) of Tropidurus hygomi (Sauria, Tropiduridae) in the Parque Nacional Serra de Itabaiana, Sergipe state, Brazil. 
predação (Paulissen 1988, Irschick et al. 2000), principalmente para espécies territorialista (Martins et al. 2018) e com comportamento de canibalismo, como registrado para T. hygomi (Kohlsdorf et al. 2004).

Estudos relacionados ao uso de microhabitats por jovens e adultos é pouco documentado na literatura para o gênero Tropidurus. Em um estudo prévio com a espécie T. melanopleurus, os jovens e adultos diferiram em relação ao uso do habitat (Perez-Mellado \& Riva 1993). Segundo estes autores essa diferença pode ser resultado da competição intraespecífica, o que diminui as chances de seleção de microhabitats para os jovens. Entretanto, existe um número substancial de trabalhos que trata as diferenças no uso de microhabitat para machos e fêmeas de Tropidurus, e para espécies simpátricas e sintópicas (e.g., Vitt et al. 1997, Cruz 1998, Faria \& Araujo 2004, Van Sluys et al. 2004, Meira et al. 2007, Ribeiro \& Freire 2011, Santana et al. 2011, Santana et al. 2014, Caldas et al.2015).

Tropidurus hygomi apresentou recrutamento durante todos os meses, com diferentes abundâncias ao longo do ano, que possivelmente implica em uma reprodução contínua com variação, como definido por Sherbrooke (1975). Essa informação corrobora os resultados histológicos apresentados por Lima (2016) para a mesma espécie na mesma área. No entanto, estudos realizados com espécies congenéricas ( $T$. torquatus, T. itambere e T. hispidus) demonstraram que em ambientes sazonais estes possuem um ciclo reprodutivo sazonal com período de recrutamento de jovens em uma determinada época do ano (Van Sluys 1993, Wiederhecker et al. 2002, Ribeiro et al. 2012).

O clima é a condição ambiental mais frequentemente associada à variação nas estratégias reprodutivas de lagartos (Dunham et al. 1988). Posto que, em regiões tropicais, onde a precipitação é bem distribuída ao longo do ano (e.g., Floresta Amazônica), algumas espécies se reproduzem continuamente com várias ninhadas por estação reprodutiva (Mesquita \& Colli 2010). Diferentemente do que ocorre em regiões com clima sazonal (e.g., Caatinga) onde as espécies se reproduzem sazonalmente com apenas uma ou algumas ninhadas por estação reprodutiva (Vitt 1983, Wiederhecker et al. 2002, Mesquita \& Colli 2010).
A relação dos adultos de T. hygomi com a pluviosidade neste estudo pode estar relacionado com o maior investimento da sua reprodução nesse período. Apesar de não ter uma relação entre a abundância de jovens e pluviosidade, é notório que em alguns meses chuvosos a sua abundância foi relativamente alta, o que pode estar associado às condições favoráveis da chuva. Em espécies do gênero Tropidurus, aparentemente existe uma tendência à concentração de atividades reprodutivas durante a estação chuvosa, em diferentes regiões (Wiederhecker et al. 2002, Ribeiro et al. 2012). Logo, à presença de condições microclimáticas favoráveis na estação chuvosa favorece o desenvolvimento embrionário e eclosão dos ovos, e a sobrevivência da prole, pois fornece uma maior disponibilidade de alimentos (Ribeiro et al. 2012).

Ambientes úmidos são importantes no desenvolvimento dos ovos, pois a umidade influencia no sucesso de incubação (James \& Shine 1985), evitando a dessecação dos embriões e dos filhotes (Sexton et al. 1971, Packard et al. 1982, Galdino et al. 2003). Sendo assim, a relação dos adultos de T. hygomi com a precipitação pode ser de fundamental importância, principalmente, por ocuparem ambientes abertos, que são mais propensos ao estresse da perda de água.

Nossos dados sugerem que os jovens e os adultos do lagarto T. hygomi não diferiram quanto ao uso do microhabitat. Apresentaram um período de recrutamento contínuo, mas com uma leve variação ao longo das estações. Acreditamos que a sobreposição encontrada entre as faixas etárias esteja relacionada à qualidade dos habitats, com áreas mais sombreadas para evitar o superaquecimento.

\section{AGRADECIMENTOS}

Os autores agradecem ao ICMBio por ceder o alojamento do Parque Nacional Serra de Itabaiana durante as atividades de campo. À Universidade Federal de Sergipe por disponibilizar transporte para o desenvolvimento da pesquisa. A toda a equipe do Laboratório de Biologia e Ecologia de Vertebrados (LABEV) pelo apoio durante as atividades de campo. Para o presente trabalho foi obtida a licença para atividades científicas (SISBIO / ICMBio, $n^{\circ} 45723-1$ ). 


\section{REFERÊNCIAS}

Balestrin, R. L., Cappellari, L. H., \& Outeiral, A. B. 2010. Biologia reprodutiva de Cercosaura schreibersii (Squamata, Gymnophthalmidae) e Cnemidophorus lacertoides (Squamata, Teiidae) no Escudo Sul-Riograndense, Brasil. Biota Neotropica, 10(1), 131-139. DOI: 10.1590/ S1676-06032010000100013

Bezerra, M. F. A., Lado, C., \& Cavalcanti, L. H. 2007. Mixobiota do Parque Nacional Serra de Itabaiana, SE, Brasil: Liceales. Acta de Botânica Brasílica, 21(1), 107-118. DOI: 10.1590/S010233062010000200021

Caldas, F. L. S., Santana, D. O., Santos, R. A., Gomes, F. F. A., Silva, B. D., \& Faria, R. G. 2015. Atividade e uso do espaço de Tropidurus semitaeniatus (Iguania) em área de Mata Atlântica, Nordeste do Brasil. Neotropical Biology and Conservation, 10(2), 85-92. DOI: 10.4013/nbc.2015.102.04

Carvalho, A. L. G. 2013. On the distribution and conservation of the South American lizard genus Tropidurus Wied-Neuwied, 1825 (Squamata: Tropiduridae). Zootaxa, 3640(1), 42-56. DOI: 10.11646/zootaxa.3640.1.3

Colli, G. R., Araujo, A. F. B., Silveira, R., \& Roma, F. 1992. Niche partitioning and morphology of two syntopic Tropidurus (Sauria: Tropiduridae) in Mato Grosso, Brazil. Journal of Herpetology, 26(1), 66-69. DOI: 10.2307/1565023

Cruz, F. B. 1998. Ecology of the lizard Tropidurus etheridgei (Squamata: Tropiduridae) from the dry Chaco of Salta, Argentina. Herpetological Natural History, 6(1), 23-31.

Curto, R. A., Silva, G. F., Soares, C. P. B., Martins, L. T., \& David, H. C. 2013. Métodos de estimação de altura de árvores em Floresta Estacional Semidecidual. Floresta, 43(1), 105-116. DOI: 10.5380/rf.v43i1.26791

Dantas, T. V. P., \& Ribeiro, A. S. 2010. Caracterização da vegetação do Parque Nacional Serra de Itabaiana, Sergipe - Brasil. Biotemas, 23(4), 9-18. DOI: 10.5007/2175-7925.2010v23n4p9

Dantas, T. V. P., Nascimento-Júnior, J. E., Ribeiro, A. S., \& Prata, A. P. N. 2010. Florística e estrutura da vegetação arbustivo-arbórea das Areias Brancas do Parque Nacional Serra de Itabaiana/Sergipe, Brasil. Revista Brasileira de Botânica, 33(4), 575588. DOI: $10.1590 / \mathrm{s} 0100-84042010000400006$

Dias, E. J. R., \& Rocha, C. F. D. 2014. Habitat structural effect on Squamata fauna of the Restinga ecosystem in Northeastern Brazil. Anais da Academia Brasileira de Ciências, 86(1), 359-371. DOI: 10.1590/0001-3765201420130006

Dunham, A. E., Miles, D. B., \& Reznick, D. N. 1988. Life history patterns in squamate reptiles. In: C. Gans, \& R. B. Huey (Eds.), Biology of the Reptilia: Ecology B. Defense and Life History. pp. 441522. New York: Alan R. Liss, Inc.

Faria, R. G., \& Araujo, A. F. B. 2004. Sintopy of two Tropidurus lizard species (Squamata: Tropiduridae) in a rocky Cerrado habitat in Central Brazil. Brazilian Journal of Biology, 64(4), 775-786. DOI: $10.1590 / \mathrm{s} 1519$ 69842004000500007

Galdino, C. A. B., Assis, V. B., Kiefer, M. C., \& Van Sluys, M. 2003. Reproduction and fat body cycle of Eurolophosaurus nanuzae (Sauria; Tropiduridae) from a seasonal montane habitat of Southeastern Brazil. Journal of Herpetology, 37(4), 687-694. DOI: 10.1670/114-02A

Gibbons, J. R. H., \& Lillywhite, H. B. 1981. Ecological segregation, color matching, and speciation in lizards of the Amphibolurus decresii species complex (Lacertilia: Agamidae). Ecology, 62(6), 1573-1584. DOI: 10.2307/1941513

Grover, M. C. 1996. Microhabitat use and thermal ecology of two narrowly sympatric Sceloporus (Phrynosomatidae) lizards. Journal of Herpetology, 30(2), 152-160.

Hatano, F. H., Vrcibradic, D., Galdino, C. A. B., Cunha-Barros, M., Rocha, C. F. D., \& Van Sluys, M. 2001. Thermal ecology and activity patterns of the lizard community of the restinga of Jurubatiba, Macaé, RJ. Revista Brasileira de Biologia, 61(2), 287-294. DOI: 10.1590/S003471082001000200011

IBGE. 1992. Manual técnico da vegetação brasileira: Manuais Técnicos em Geociências número 1. Rio de Janeiro, Brasil, 94 pp. Acesso em 25/02/2019 em https://biblioteca.ibge.gov.br/ visualizacao/livros/liv63011.pdf

Irschick, D. J., Macrini, T. E., Koruba, S., \& Forman, J. 2000. Ontogenetic differences in morphology, habitat use, behavior, and sprinting capacity in two West Indian Anolis lizards. Journal of Herpetology, 34(3), 444-451. DOI: $10.2307 / 1565368$

James, C., \& Shine, R. 1985. The seasonal timing of reproduction: A Tropical-Temperate 
comparison in Australian lizards. Oecologia, 67(4), 464-474. DOI: 10.1007/BF00790016

Kohlsdorf, T., Godoy, C., \& Navas, C. A. 2004. Tropidurus hygomi (NCN): Cannibalism. Herpetological Review, 35, 398.

Lima, J. O. 2016. Efeitos de gradientes ambientais sobre a distribuição espacial, atividade, dieta e reprodução de lagartos do gênero Tropidurus (Squamata:Tropiduridae) na Serra de Itabaiana, Sergipe. Programa de Pós-Graduação em Diversidade Animal da Universidade Federal da Bahia. p. 64.

Marcellini, D., \& Mackey, J. P. 1970. Habitat preferences of the lizards, Sceloporus occidentalis and S. graciosus (Lacertilia, Iguanidae). Herpetologica, 26(1), 51-56.

Martins, K. V., Dias, E. J. R., \& Rocha, C. F. D. 2010. Ecologia e conservação do lagarto endêmico Tropidurus hygomi (Sauria: Tropiduridae) nas restingas do Litoral Norte da Bahia, Brasil. Biotemas, 23(4), 71-75. DOI: 10.5007/2175-7925.2010v23n4p71

Martins, K. V., Andrade, H., \& Dias, E. J. R. 2018. Efeito do ambiente térmico na atividade diária de Tropidurus hygomi (Squamata: Tropiduridae) em restingas do Nordeste do Brasil. Oecologia Australis, 22(1), 17-27. DOI: 10.4257/oeco.2018.2201.02

Meira, K. T. R., Faria, R. G., Silva, M. D. M., Miranda, V. T., \& Zahn-Silva, W. 2007. História natural de Tropidurus oreadicus em uma área de cerrado rupestre do Brasil Central. Biota Neotropica, 7(2), 155-163.

Mesquita, D. O., \& Colli, G. R. 2010. Life history patterns in Tropical South American lizards. In: O. H. Gallegos, F. R. M. Cruz \& J. F. M. Sánchez (Eds.), Reproducción en reptiles: Morfología, ecología y evolución. pp. 47-71. Toluca: Universidad Autónoma del Estado de México.

MMA. 2016. Ministério do Meio Ambiente. Livro Vermelho da Fauna Brasileira Ameaçada de Extinção. Acesso em 12/01/2019 em http:// www.icmbio.gov.br/portal/images/stories/ comunicacao/publicacoes/publicacoesdiversas/dcom_sumario_executivo_livro_ vermelho_ed_2016.pdf

Packard, M. J., Packard, G. C., \& Boardman, T.J. 1982. Structure of eggshells and water relations of reptilian eggs. Herpetologica, 38(1), 136-155.

Paulissen, M. A. 1988. Ontogenetic and seasonal shifts in microhabitat use by the lizard Cnemidophorus sexlineatus. Copeia, 1988(4), 1021-1029. DOI: 10.2307/1445727

Peel, M. C., Finlayson, B. L., \& Mcmahon. 2007. Updated world map of the Köppen-Geiger climate classification. Hydrology and Earth System Sciences Discussions, 11(5), 1633-1644.

Perez-Mellado, V., \& Riva, I. 1993. Sexual size dimorphism and ecology: The case of a Tropical lizard, Tropidurus melanopleurus (Sauria: Tropiduridae). Copeia, 1993(4), 969-976.

Pianka, E. R. 1986. Ecology and natural history of desert lizards: analyses of the ecological niche and community structure. New Jersey: Princeton University Press: p. 208.

R Development Core Team. 2017. R: A language and environment for statistical computing. Viena, Austria: R Foundation for Statistical Computing. Available from www.R-project.org.

Ribeiro, L. B., \& Freire, E. M. X. 2011. Trophic ecology and foraging behavior of Tropidurus hispidus and Tropidurus semitaeniatus (Squamata, Tropiduridae) in a caatinga area of northeastern Brazil. Iheringia, 101(3), 225-232. DOI: $10.1590 / S 0073-47212011000200010$

Ribeiro, L. B., Silva, N. B., \& Freire, E. M. X. 2012. Reprodutive and fat body cycles of Tropidurus hispidus and Tropidurus semitaeniatus (Squamata, Tropiduridae) in a caatinga area of northeastern Brazil. Revista Chilena de Historia Natural, 85(3), 307-320.

Rocha, C. F. D. 1992. Reproductive and fat body cycles of the Tropical Sand Lizard (Liolaemus lutzae) of Southeastern Brazil. Journal of Herpetology, 26(1), 17-23. DOI: 10.2307/1565016

Rocha, C. F. D. 1998. Population dynamics of the endemic tropidurid lizard Liolaemus lutzae in a seasonal restinga habitat. Ciência e Cultura Journal of the Brazilian Association for the Advancement of Science, 50(6), 446-451.

Rocha, C. F. D., Vrcibradic, D., \& Araújo, A. F. B. 2000. Ecofisiologia de Répteis de Restingas Brasileiras. In: F. A. Esteves \& L. C. Lacerda (Eds.), Ecologia de Restingas e Lagoas Costeiras. pp. 117-149. Macaé, Rio de Janeiro: NUPEM/ UFRJ.

Rodrigues, M. T. 1988. Distribution of lizards of the genus Tropidurus in Brazil (Sauria, Iguanidae). In: W. R. Heyer \& P. E. Vanzolini (Eds.), Proceedings of a Workshop on Neotropical 
Distribution Patterns. pp. 305-315. Rio de Janeiro: Academia Brasileira de Ciências.

Salzburg, M. A. 1984. Anolis sagrei and Anolis cristatellus in Southern Florida: A case study in interspecific competition. Ecology, 65(1), 14-19. DOI: $10.2307 / 1939453$

Santana, D. O., Faria, R. G., Ribeiro, A. S., Oliveira, A. C. F., Souza, B. B., Oliveira, D. G., Santos, E. D., Soares, F. A. M., Gonçalves, F. B., Calasans, H. C. M., Vieira, H. S., Cavalcante, J. G., Marteis, L. S., Aschoff, L. C., Rodrigues, L. C., Xavier, M. C. T., Santana, M. M., Soares, N. M., Figueiredo, P. M. F. G., Barretto, S. S. B., Franco, S. C., \& Rocha, S. M. 2011. Utilização do microhábitat e comportamento de duas espécies de lagartos do gênero Tropidurus numa área de Caatinga no Monumento Natural Grota do Angico. Scientia Plena, 7(4), 01-09.

Santana, D. O., Caldas, F. L. S., Gomes, F. F. A., Santos, R. A., Silva, B. D., Rocha, S. M., \& Faria, R. G. 2014. Aspectos da história natural de Tropidurus hispidus (Squamata: Iguania: Tropiduridae) em área de Mata Atlântica, nordeste do Brasil. Neotropical Biology and Conservation, 9(1), 55-61. DOI: 10.4013/ nbc.2014.91.07

Shapiro, S. S., \& Wilk, M. B. 1965. An analysis of variance test for normality (complete samples). Biometrika, 52, 591-611.

Sexton, O. J., Ortleb, E. P., Hathaway, L. M., Ballinger, R. E., \& Licht, P. 1971. Reproductive cycles of three species of anoline lizards from the Isthmus of Panama. Ecology, 52(2), 201-215. DOI: $10.2307 / 1934579$

Sherbrooke, W. C. 1975. Reproductive cycle of a tropical teiid lizard, Neusticurus ecpleopus Cope, in Peru. Biotropica, 7(3), 194-207. DOI: $10.2307 / 2989623$

Simpson, E. H. 1949. Measurement of diversity. Nature, 163, 688.

Teixeira, R. L., \& Giovanelli, M. 1999. Ecologia de Tropidurus torquatus (Sauria: Tropiduridae) da restinga de Guriri, São Mateus, ES. Revista Brasileira de Biologia, 59(1), 11-18. DOI: 10.1590/ S0034-71081999000100002

Vargens, M. M. F., Dias, E. J. R., \& Lira-da-Silva, R. M. 2008. Ecologia Térmica, período de atividade e uso de micro-habitat de lagarto Tropidurus hygomi (Tropiduridae) na restinga de Abaeté, Salvador, Bahia, Brasil. Boletim do Museu de
Biologia Mello Leitão, 23, 143-156.

Van Sluys, M. 1993. The reproductive cycle of Tropidurus itambere (Sauria: Tropiduridae) in Southeastern Brazil. Journal of Herpetology, 27(1), 28-32. DOI: 10.2307/1564901

Van Sluys, M. 2000. Population dynamics of the saxicolous lizard Tropidurus itambere (Tropiduridae) in a seasonal habitat of southeastern Brazil. Herpetologica, 56(1), 5562.

Van Sluys, M., Rocha, C. F. D., Vrcibradic, D., Galdino, C. A. B., \& Fontes, A. F. 2004. Diet, activity, and microhabitat use of two syntopic Tropidurus species (Lacertilia: Tropiduridae) in Minas Gerais, Brazil. Journal of Herpetology, 38(4), 606-611. DOI: 10.1670/218-03N

Vieira, R. C., Felappi, J. F., Caruccio, R., \& Verrastro, L. 2011. Population dynamics of Tropidurus torquatus (Wied, 1820) (Squamata, Tropiduridae) in Southern Brazil. Journal of Herpetology, 6(3), 215-222. DOI: 10.2994/057.006.0308

Vitt, L. J. 1983. Reproduction and sexual dimorphism in the Tropical teiid lizard Cnemidophorus ocellifer. Copeia, 1983(2), 359366. DOI: $10.2307 / 1444378$

Vitt, L. J. 1991. Ecology and life history of the scansorial arboreal lizard Plica plica (Iguanidae) in Amazonian Brazil. Canadian Journal of Zoology, 69(2), 504-511. DOI: 10.1139/ z91-077

Vitt, L. J. 1993. Ecology of isolated open-formation Tropidurus (Reptilia: Tropiduridae) in Amazonian lowland rain forest. Canadian Journal of Zoology, 71(12), 2370-2390. DOI: 10.1139/z93-333

Vitt, L.J.,Zani, P.A., \&Avila-Pires, T.C. 1997.Ecology of the arboreal tropidurid lizard Tropidurus (= Plica) umbra in the Amazon region. Canadian Journal of Zoology, 75(11), 1876-1882.

Wiederhecker, H. C., Pinto, A. C. S., \& Colli, G. R. 2002. Reproductive ecology of Tropidurus torquatus (Squamata: Tropiduridae) in the highly seasonal Cerrado biome of Central Brazil. Journal of Herpetology, 36(1), 82-91. DOI: 70/0022-1511(2002)036[0082:REOTTS]2.0.CO;2

Xavier, M. A., \& Dias, E. J. R. 2015. First record of the Brazilian restinga lizard Tropidurus hygomi ingesting a fruit of Melocactus violaceus (Cactaceae). Herpetology Note, 8, 437-438. 
Xavier, M. A., \& Dias, E. J. R. 2017. Ecology of endemic and saxicolous lizard Tropidurus pinima (Rodrigues, 1984) from Caatinga domain, Brazil. Herpetology Notes, 10, 491-492.

Zar, J. H. 2010. Biostatistical Analysis. 5 nd. ed. New Jersey: Prentice Hall press: p. 944.

Submetido em: 10/10/2018

Aceito em: 16/05/2019

Publicado online: 15/06/2019

Editor Associado: Leandro dos Santos Lima Hohl 